\title{
The impact of the lung environment on macrophage development, activation and function: diversity in the face of adversity
}

\author{
Calum C. Bain ${ }^{1,3 凶}$ and Andrew S. MacDonald ${ }^{2,3 凶}$ \\ (c) The Author(s) 2022
}

The last decade has been somewhat of a renaissance period for the field of macrophage biology. This renewed interest, combined with the advent of new technologies and development of novel model systems to assess different facets of macrophage biology, has led to major advances in our understanding of the diverse roles macrophages play in health, inflammation, infection and repair, and the dominance of tissue environments in influencing all of these areas. Here, we discuss recent developments in our understanding of lung macrophage heterogeneity, ontogeny, metabolism and function in the context of health and disease, and highlight core conceptual advances and key unanswered questions that we believe should be focus of work in the coming years.

Mucosal Immunology (2022) 15:223-234; https://doi.org/10.1038/s41385-021-00480-w

\section{INTRODUCTION}

Macrophages are largely sessile, tissue dwelling phagocytes that are present in every organ of the body. The biology of macrophages has been studied for well over one hundred years since being described by Elie Metchnikoff at the end of the nineteenth century. However, arguably, it is the last 10-15 years that has seen major revisions of key concepts in macrophage biology, from nomenclature of subsets and activation states to the ontogeny of these cells. For instance, while macrophages have historically been described as M1 ("classically-activated") or M2 ("alternatively-activated") largely based on in vitro culture systems ${ }^{1}$, it is now beyond doubt that a binary classification such as this is inadequate to capture the complexity of macrophage plasticity and activation states, particularly in vivo ${ }^{2}$. Technological advances have revealed tremendous diversity and heterogeneity between macrophages from different tissues and even within different niches of the same tissue in terms of phenotype, transcriptome and metabolome ${ }^{3,4}$. While macrophages generally excel at the 'silent' clearance of debris, apoptotic host cells and the capture and destruction of microbial intruders, it is now clear they also play far broader roles and are exquisitely tailored to meet the demands of the local tissue microenvironment in which they reside. Indeed, the in vivo tissue environment may be the major determinant governing macrophage development, recruitment, activation and function, highlighting the importance of careful consideration of the distinctive properties that different tissues possess, in steady state and during inflammation, to fully understand the role of macrophages in different locations throughout the body.

In this article, we review recent developments in the understanding of macrophage heterogeneity, ontogeny and function in lung health and during inflammation, immunity and tissue repair. Using pulmonary fibrosis as an example, we discuss how dysregulated macrophage behaviour can contribute to lung pathology and how mouse models have revealed the complexity of the macrophage response to lung injury. Along the way we identify key areas that we believe warrant further investigation.

\section{DEFINING PULMONARY MACROPHAGES IN THE HEALTHY LUNG}

Macrophages are distributed throughout the lung and can broadly be divided into those present in the airways/alveoli and those in the tissue interstitium/parenchyma. While it has been clear for decades that pulmonary macrophages exist in both airways and tissues, it is only recently that we have come to appreciate the degree of heterogeneity and diversity between different macrophage subsets in each location. In particular, the advent of single cell technologies, such as single cell RNA sequencing (scRNA-seq), has allowed tissue macrophage heterogeneity to be assessed in a completely unbiased manner across several species ${ }^{5-11}$.

In mice, alveolar macrophages (AlvMфs; see Box 1) in the healthy lung are defined by their high and uniform expression of CD11C, SiglecF and CD169 (Siglec1; sialoadhesin), and lack of CD11b expression ${ }^{12-14}$. Their residence in the airways can be confirmed by performing bronchoalveolar lavage (BAL) where they are the sole macrophage population in health ${ }^{15}$, although it is important to note that this method only retrieves a fraction of the macrophages resident in the airways. Murine interstitial macrophages (IntM $\phi s$ ) express high levels of $\mathrm{CD} 11 \mathrm{~b}$, but lack expression of SiglecF ${ }^{12-14}$. Compared with AlvM $\phi s$, considerably less is known about the IntM $\phi$ compartment, likely reflecting the fact that these macrophages are

\footnotetext{
${ }^{1}$ The University of Edinburgh Centre for Inflammation Research, Queen's Medical Research Institute, Edinburgh Bioquarter, Edinburgh EH16 4TJ, UK. ${ }^{2}$ Lydia Becker Institute of Immunology and Inflammation, University of Manchester, Manchester M13 9NT, UK. ${ }^{3}$ These authors contributed equally: Calum C. Bain, Andrew S. MacDonald.

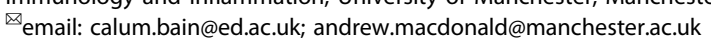


Box 1. What's in a name?

In order to discuss pulmonary macrophage subsets, some clarity on nomenclature is needed. Historically, macrophages found in the airways/bronchoalveolar lavage fluid (BALF) of naïve mice have been termed 'alveolar macrophages', which express high levels of $C D 11 c$, SiglecF and CD169 while lacking CD11b expression. These airway/alveolar macrophages are phenotypically and transcriptionally distinct from those found in the lung tissue, which express CD11b and variable levels of CD11c, but lack SiglecF expression and are often termed as 'lung tissue', 'interstitial' or 'parenchymal' macrophages. It is now clear that, during inflammation, macrophages with a tissue/interstitial phenotype can be readily found in the airways/BALF. Thus, for clarity, in this review we will use 'alveolar' only to refer to airway macrophages (AM $\phi s)$ that are $C D 11 c^{\text {hi }}$ SiglecF ${ }^{+}$(AlvM $\left.\phi s\right)$, and 'interstitial' to refer to $\mathrm{CD}_{11 \mathrm{~b}^{+} \text {SiglecF }}$ lung tissue macrophages (IntM $\left.\phi s\right)$. We will use the term monocyte-derived AMфs to refer to macrophages in the BALF that have been shown to arise in the context of inflammation and exhibit some, but not all, features of bona fide AlvM $\phi s$. We will use the term 'ex-interstitial' (ex-IntM $\phi$ ) to try to make clear when we are referring to cases where macrophages with this phenotype are found in the airways. Since it is currently impossible to distinguish tissue vs airway origins of human pulmonary macrophages, we will simply refer to cells obtained by BAL as airway macrophages (AM $\phi S$ ) and those obtained from human lung tissue as tissue macrophages (tissue Mфs).

difficult to isolate from lung tissue using standard enzymatic protocols. Indeed, flow cytometric analysis of whole mouse lung digests suggests that IntM $\phi$ s form a small fraction of the overall macrophage compartment in health, with AlvMфs outnumbering them by $5-10$-fold ${ }^{15,16}$. Whether this is also the case in humans is currently hard to determine with certainty, as no comparable set of surface markers has yet been identified to unequivocally distinguish human AlvM $\phi$ s from IntM $\phi s$ in BAL, sputum or in lung tissue digests. Nevertheless, limited fluorescence microscopy of mouse and human lung, which circumvents the need to dissociate solid tissue, suggests that tissue (IntM $\phi$ s) are more abundant than suggested by flow cytometry ${ }^{17-19}$. Thus, the notion that these cells represent a minor macrophage population should be reconsidered (Box 2).

It is also important to note that due to the overlapping expression of CD11c, CD11b and SiglecF by other myeloid cells, in particular $\mathrm{CD}_{11 \mathrm{c}^{+}}$dendritic cells (DCs) and SiglecF $^{+} \mathrm{CD} 11 \mathrm{~b}^{+}$ eosinophils, expression of these surface markers alone is not sufficient for the characterisation of murine lung macrophages. Instead, a more rigorous approach is needed to define bona fide murine pulmonary macrophage subsets, with a growing consensus that the optimal strategy is by their co-expression of the high affinity FcyR1 (CD64) and Mer-tyrosine kinase (MerTK), a key efferocytic receptor ${ }^{12,15,20}$. In health, AlvM $\phi s$ and IntM $\phi$ s can then be identified amongst the $\mathrm{CD}^{+} 4^{+} \mathrm{MerTK}^{+}$fraction by their distinct $\mathrm{CD} 11 \mathrm{c} / \mathrm{CD} 11 \mathrm{~b}$ profiles and other phenotypic traits (Fig. 1). As discussed below, distinction between these anatomically distinct cells becomes less apparent when homeostasis is perturbed. Moreover, recent work has shown that some DCs can acquire expression of CD64 in certain contexts ${ }^{21}$, emphasising the need for multi-parameter analysis when characterising these cells. Notably, neither AlvMфs nor IntM $\phi$ s can be defined using the M1/M2 nomenclature system. Indeed, in the healthy lung both populations co-express markers historically considered " $\mathrm{M} 1$ " and "M2" specific 14,15. For instance, murine AlvMфs constitutively coexpress CD11c and $\mathrm{Ym}^{-1}$ (encoded by Chil3) which have been used by some as defining features of so-called "M1" and "M2" macrophages ${ }^{22,23}$. However, Ym-1 expression by homeostatic AlvM $\phi s$ is independent of IL-4-IL-4R signalling ${ }^{15}$, the axis controlling "alternative" activation of macrophages, and CD11c expression is independent of exposure to microbial products or inflammatory cytokines thought to drive so-called " $\mathrm{M} 1$ " polarisation $^{24,25}$. This highlights the inadequate nature of the M1/M2 nomenclature in defining macrophages in vivo and that as a field we need to abandon using $\mathrm{it}^{26}$.

Recent studies employing scRNA-seq have begun to reveal additional heterogeneity in the murine pulmonary macrophage compartment. Whereas AlvM $\phi$ s appear to be relatively
Box 2. Key Challenges

1. Establish "signatures" to faithfully distinguish human AlvMфs from IntMфs and ex-IntM $\phi s$

2. Develop methods of lineage tracing in human lung macrophage subsets

3. Reduce reliance on in vitro functional assays of questionable relevance and develop models/systems/approaches that better represent the lung environment

4. Reach consensus on the heterogeneity, function and spatial distribution of IntMфs

5. Develop novel transgenic murine models to selectively target AlvMфs vs IntM $\phi s$ vs ex-IntM $\phi$ s and understand the molecular levers that control their differentiation

6. Transition to using mouse models with microbial exposure/status more aligned with that encountered by humans.

homogeneous ${ }^{6}$, the $\operatorname{IntM} \phi$ compartment harbours at least two distinct subsets defined by their expression of CD206 (mannose receptor) ${ }^{14,18,27}$. The anatomical locale in which each of these subsets are found remains contentious. $\mathrm{CD}^{206^{-}}$IntM $\phi$ s that mostly express $\mathrm{MHCl}^{+}$have been suggested to be enriched in the interstitial tissue adjacent to the alveoli, whereas their CD206 ${ }^{+}$ $\mathrm{MHCII}^{-}$counterparts appear more numerous in the interstitium surrounding the bronchi ${ }^{18}$. Multi-parameter fluorescence microscopy has also started to elucidate the nature of the niche in which these subsets may exist. For instance, CD206 $\mathrm{MHCll}^{+}$ IntMфs can be found to interact with nerves, whereas CD206 ${ }^{+}$ $\mathrm{MHCII}^{-}$cells may occupy a perivascular niche ${ }^{27}$. Transcriptional profiling has revealed other useful markers for the identification of these subsets, including Lyve-1, folate receptor beta (FR $\beta)$ and CD36, which appear to be more highly expressed by perivascular $\mathrm{CD}^{206}{ }^{+} \mathrm{MHCll}^{-}$macrophages. Work by the Khanna group identified a population of CD169-expressing IntM $\phi s^{28}$, although it seems these largely overlap transcriptionally with the $\mathrm{CD}^{206^{-}} \mathrm{MHCII}^{+}$IntM $\phi$ s identified by others. CX3CR1 has also been suggested as a defining marker of these subsets ${ }^{27}$, although other studies have shown that CX3CR1 is expressed equally across these subsets $^{14,18,29}$. Thus, while it is clear that the murine IntM $\phi$ compartment is heterogeneous, consensus is yet to be reached on the best strategy to define these cells.

The identity of macrophage subsets in the human lung has also become clearer recently. Human AMфs express high levels of HLADR, CD11b and CD206 together with CD169 and MARCO 7,13,30. CD163 appears to define two subsets of $A M \phi$ in lung tissue from humans and non-human primates. However, whereas CD163 $3^{\text {hi }}$ AM $\phi s$ are abundant in BALF, CD163 ${ }^{\text {lo }}$ AM $\phi s$ are relatively rare ${ }^{13}$, questioning whether they truly represent AlvMфs. Importantly, while SiglecF has become somewhat of a de facto marker of murine AlvMфs, its human paralog, Siglec8, is absent from AM $\phi s$ in $\operatorname{man}^{13}$. Despite only partial conservation in terms of phenotype between mice and humans, recent transcriptional profiling suggests that these cells share a core gene signature, including expression of PPARG, FABP4, FFAR4, FN1 1,31-34 (Fig. 1). The phenotypic and transcriptional identity of human IntM $\phi s$ during health is limited by access to healthy lung tissue. Nevertheless, they appear to be defined as $\mathrm{HLA}-\mathrm{DR}^{+} \mathrm{CD} 11 \mathrm{~b}^{+} \mathrm{CD} 36^{+}$cells lacking CD169 expression ${ }^{13}$, a phenotype that has recently been confirmed using humanised mice ${ }^{35}$. Moreover, heterogeneity similar to that seen in mice is likely to exist amongst human IntM $\phi s^{27}$, with one study suggesting the intensity of HLA-DR and CD36 a defining characteristic ${ }^{7}$.

\section{MACROPHAGE FUNCTION IN HEALTH}

As immunologists, we tend to consider the primary function of macrophages is to act as the first line of defence against pathogens. Indeed, the positioning of macrophages in the airways 


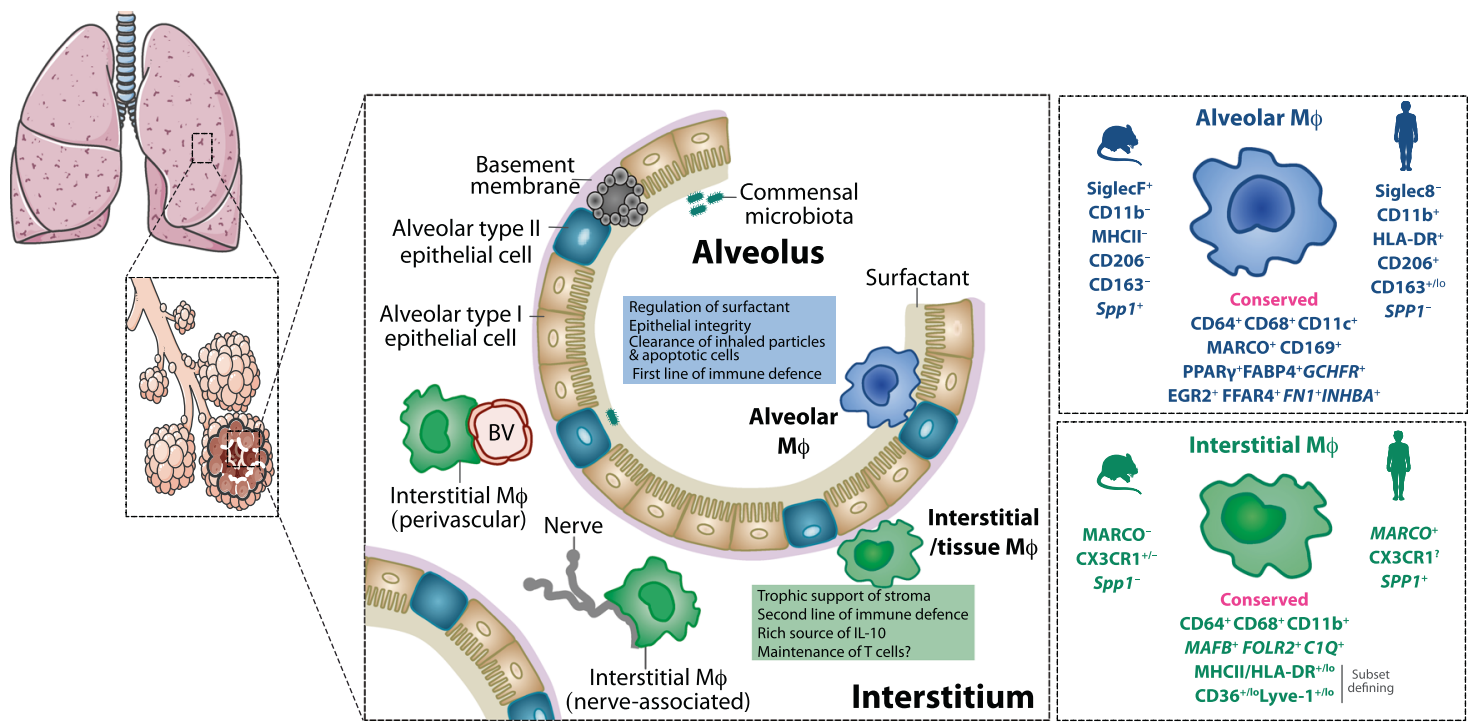

Fig. 1 Heterogeneity, phenotypic profiles and functions of macrophages in the healthy lung. The lung macrophage compartment is heterogeneous, with at least two populations occupying distinct anatomical niches in the healthy lung. Macrophages are present in the bronchoalveolar space, including the alveoli where gaseous exchange occurs. Alveolar macrophages (AlvM $\phi s$ ) are defined by their expression of CD11C, MARCO and CD169 in both mice and humans, although additional species-specific markers must be used to define them accurately. AlvM $\phi s$ are crucial for regulating surfactant produced by the respiratory epithelium as well as maintaining epithelial integrity and responsiveness. Their high phagocytic capability allows them to clear apoptotic/senescent cells and inhaled particles efficiently. They also act as the first line of defence against air-borne pathogens, although the relative role of resident AlvM $\phi s$ versus elicited, monocyte-derived macrophages in immune protection varies depending on the nature of the insult (see text and Fig. 3). Macrophages are also found in the interstitial space between the alveoli and the capillary beds, as well as surrounding larger airways (bronchi). These interstitial macrophages (IntMфs) are phenotypically distinct from AlvMфs and at least two subsets exist in mouse and man defined by differential expression of $\mathrm{MHCII}$ (HLA-DR), Lyve-1 and/or CD36. IntMфs may act as a second line of defence under the epithelial barrier and basement membrane. In health, they may support the stromal/structural compartment through growth factor supply, as well as maintaining T cells and acting a rich source of IL-10. Although nerve- and blood vessel (BV)-associated IntM $\phi s$ have been described, whether these represent obligate niches is under debate.

means that they will be one of the first cells to encounter airborne pathogens and altering the ability of airway macrophages to detect, engulf and kill respiratory pathogens leads to increased susceptibility to a variety of bacteria, viruses and fungi ${ }^{36-41}$. However, as described below, to avoid excessive and potentially harmful pro-inflammatory responses being mounted against environmental antigens encountered by airway macrophages, these cells are held in a state of relative hyporesponsiveness via a range of powerful inhibitory mechanisms ${ }^{42}$. In the absence of infection, the principal role of airway macrophages is the regulation of pulmonary surfactant, the lipid-protein complex produced by the respiratory epithelium to lubricate the lungs and allow frictionless expansion/contraction. Consistently, the AlvM $\phi$ transcriptional signature is dominated by genes associated with lipid metabolism in mouse and $\operatorname{man}^{7,43,44}$. Macrophages are indispensable for this function, as spontaneous pulmonary alveolar proteinosis (PAP) develops in mice and humans with absent or dysfunctional AlvM $\phi \mathrm{s}^{45-50}$. Likewise, dead, dying and senescent cells accumulate in the absence of functional airway macrophages, demonstrating their key efferocytic role ${ }^{43}$. Airway macrophages may also maintain the integrity and responsiveness of the respiratory epithelium. For instance, production of immunoregulatory cytokines, such as TGF $\beta$ and IL-10, may modify epithelial cell function through regulation of ion and fluid transport $^{42,51}$ and AlvM $\phi$-derived fibronectin may act as a proliferative factor for airway epithelial cells ${ }^{52}$. Suppressor of cytokine signalling (SOCS)-containing vesicles released from AlvMфs may regulate the responsiveness of the epithelium to e.g., TLR ligands ${ }^{53,54}$.

Compared with AlvM $\phi s$, the mechanisms of regulation and homeostatic functions of IntM $\phi$ s are poorly understood ${ }^{55}$, although it is clear that all murine IntM $\phi$ subsets are avidly phagocytic and can capture E.coli bioparticles in vivo ${ }^{14,18}$, suggesting they may act as a second line of defence should the epithelial barrier be breached. Their constitutive production of IL-10 under normal physiological conditions, in both mouse and man ${ }^{18,29,56-58}$, suggests an immunoregulatory role. It is likely this involves supporting regulatory $T$ cells locally in the lung parenchyma, given that IntM $\phi s$ are thought to be non-migratory ${ }^{59}$. However, IntM $\phi$ s may also alter $T$ cell responses indirectly through IL-10-dependent modulation of DC migration and priming activity ${ }^{56}$.

Exposure to bacterial CpG DNA leads to IntM $\phi$ expansion and augmentation of IL-10 production ${ }^{29}$, suggesting these cells may be inherently anti-inflammatory. Indeed, Cx3cr1-mediated deletion of II10 leads to increases susceptibility to allergic asthma in mice ${ }^{60}$. Production of platelet-derived growth factor (PDGF) by IntM $\phi$ s suggests they may also support fibroblast and epithelial homeostasis $^{61}$. Finally, given their occupation of distinct anatomical niches, it is intriguing to speculate they may differentially contribute to nerve and vascular endothelial cell homeostasis, similar to their counterparts in the gut wall ${ }^{62}$.

\section{LUNG MACROPHAGE ONTOGENY Macrophage origins in health}

Historically, macrophages were thought to be part of a linear mononuclear phagocyte system where tissue macrophages were continually replaced by blood monocytes, which themselves are replaced by dedicated bone marrow progenitors ${ }^{63}$. However, over the last 10 years there has been a conceptual revolution in our understanding of macrophage ontogeny with the discovery that many tissue macrophages derive from embryonic progenitors and 

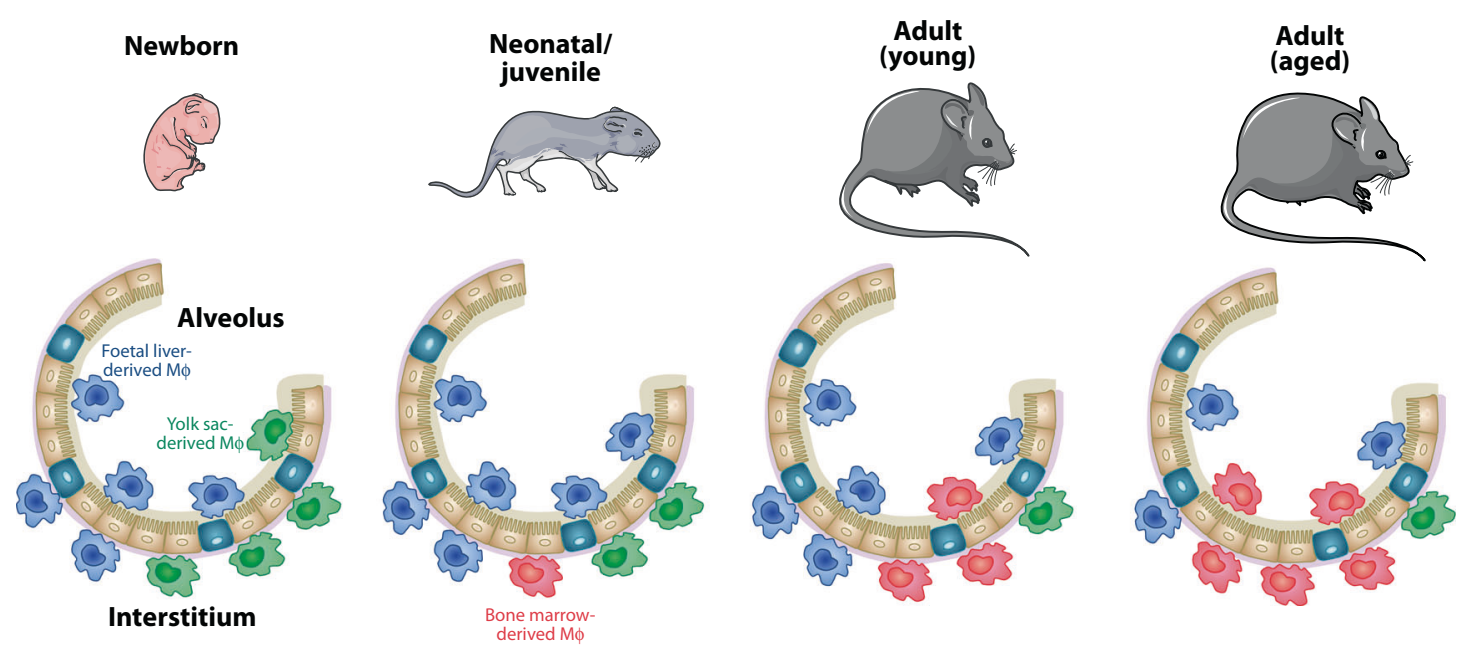

Fig. 2 Pulmonary macrophage ontogeny during health. The contribution of distinct progenitors to the pulmonary macrophage compartments is highly dynamic and alters with age. During embryonic development (in mice) yolk sac-derived macrophages colonise the lung and these remain present at birth. However, these are outnumbered by foetal liver-derived progenitors that enter the lung prior to birth, some of which move into the airways upon alveolarization within the first days of life. During the neonatal period, where there is massive tissue growth, all macrophages show high levels of proliferation to occupy the newly created niches. This is sufficient to expand the AlvM $\phi$ compartment with little, if any, contribution from bone marrow-derived monocytes. However, recent work has suggested that during adulthood under homeostatic conditions AlvM $\phi$ s are replenished, albeit at low rates, by bone marrow-derived, CCR2-dependent monocytes. These monocytes replace IntM $\phi$ s at a higher rate, although in the unperturbed lung, the IntM $\phi$ compartment likely contains macrophages derived from the yolk sac, foetal liver and bone marrow, with the latter dominating numerically.

maintain themselves autonomously through in situ self-renewal ${ }^{64-74}$. While some older studies had demonstrated the ability of macrophages to self-renew ${ }^{75}$, it is the development of elegant lineage tracing models that has led to major advances in our understanding of macrophage origins. For instance, genetic fate mapping using mice with tamoxifen-inducible Cre recombinase under the control of the Csf1r, Runx1, Cx3Cr1 or Tie2 promoters has shown that brain microglia derive from yolk sac progenitors and require little, if any, contribution from blood monocytes across the life of an animal ${ }^{64,65,67,69,76}$. Using similar systems, it was shown that yolk sac progenitors contribute minimally to lung AlvM $\phi s^{77}$. Instead, tracing of foetal and adult haematopoiesis using Flt $3^{\text {Cre }}$ mice shows haematopoietic stem cell (HSC)-derived cells make a major contribution to AlvM $\phi s^{65,68,74}$. This, combined with the fact that AlvM $\phi s$ develop within the first few days of life in mice and humans in parallel with alveolisation of the lung ${ }^{13,78,79}$, led to the idea that they derive predominantly from foetal monocytes. That AlvM $\phi s$ are unaffected in adult monocytopenic $\mathrm{CCr}^{-/-}$mice and show little exchange in the context of parabiosis or tissue protected bone marrow chimeric mice supported the notion that these cells selfmaintain throughout adult life in the absence of inflammation or infection $^{68,78}$. Moreover, analysis of AM $\phi$ longevity in the human context supported these observations in mice. By analysing macrophages obtained by transbronchial biopsies of recipients of sex mismatched lung transplants in a longitudinal manner, EguíluzGarcia et al. showed that the majority of AMфs remain of donor origin in this setting, suggesting human $A M \phi s$ maintain themselves autonomously in situ ${ }^{80}$, a finding supported by an independent study analysing AM $\phi$ s obtained by bronchoalveolar lavage ${ }^{81}$.

However, several recent studies have started to challenge this model. First, longitudinal analysis of Flt $3^{\text {Cre }}$-Rosa $26^{\text {LSL-YFP }}$ reporter mice showed increases in labelling of AlvMфs over time, indicative of age-dependent contribution of HSC-derived cells to the AlvMфpool, a phenomenon not seen in brain microglia ${ }^{65}$. This highlights the need for longitudinal analysis when considering macrophage dynamics, something that was not always performed in early lineage tracing studies ${ }^{64,74}$. Indeed, longitudinal analysis of $M s 4 a 3^{\text {Cre }}$ reporter mice, which allow tracing of all cells deriving from bone marrow granulocyte-monocyte progenitors (GMPs), supports the idea that AlvM $\phi s$ require replenishment from bone marrow-derived monocytes over the life course ${ }^{71}$ (Fig. 2). These data are consistent with recent work assessing AlvM $\phi$ turnover using so-called 'MISTRG' humanised mice, which have genes encoding human M-CSF (also known as CSF-1), GM-CSF (also known as (SF-2), IL-3 and thrombopoietin 'knocked-in' to their respective mouse loci to support human myeloid cell development, as well as a transgene encoding human SIRPa to prevent engulfment and destruction of human cells $s^{35,82}$. Moreover, the idea of AlvM $\phi$ replenishment by monocytes is supported by a recent study using scRNA-seq to determine AM $\phi$ longevity in the context of sex-mismatch lung transplants where the majority of donor $\mathrm{AM} \phi \mathrm{s}$ appear to be replaced by recipient cells ${ }^{83}$. Why different studies using transplanted tissue reach discordant conclusions is unclear, but could reflect differences in methodologies used, for instance scRNA-seq versus fluorescence in situ hybridisation (FISH) for $X / Y$ chromosomes, or the degree of injury caused by transplantation-related ischaemia and reperfusion. Clearly further work is warranted to clarify the dominant replenishment mechanisms underlying the homeostatic maintenance of AlvM $\phi s$.

The developmental origin of IntM $\phi s$ has started to be unravelled in the past few years, although these cells have attracted much less attention than their AlvM $\phi$ counterparts. Genetic fate mapping indicates an initial contribution of yolk sac progenitors to the IntM pool ${ }^{65,77}$, but these appear to be largely replaced, first by foetal liver-derived macrophages and then by HSC-derived macrophages during the early post-natal period ${ }^{27,65}$ (Fig. 2). CCR2-dependent bone marrow-derived cells continue to replenish IntM $\phi s$ during adulthood, albeit at a low rate, and despite the heterogeneity described above, IntM $\phi$ subsets appear to display similar replenishment kinetics ${ }^{27,84}$. Notably, despite clear evidence of progressive replenishment by monocytes, intact $\mathrm{CCr}^{-/-}$mice have normal numbers of IntM $\phi s^{18,29}$, serving as a cautionary note that $\mathrm{Ccr}^{-/-}$mice cannot be used in isolation to determine the contribution of monocytes to tissue macrophage pools, at least in health. This suggests potential redundancy between chemokine receptors involved in monocyte navigation ${ }^{85}$ and/or that compensatory mechanisms maintain macrophages in the context of monocytopenia. Indeed, both AlvM $\phi s$ and IntM $\phi s$ 

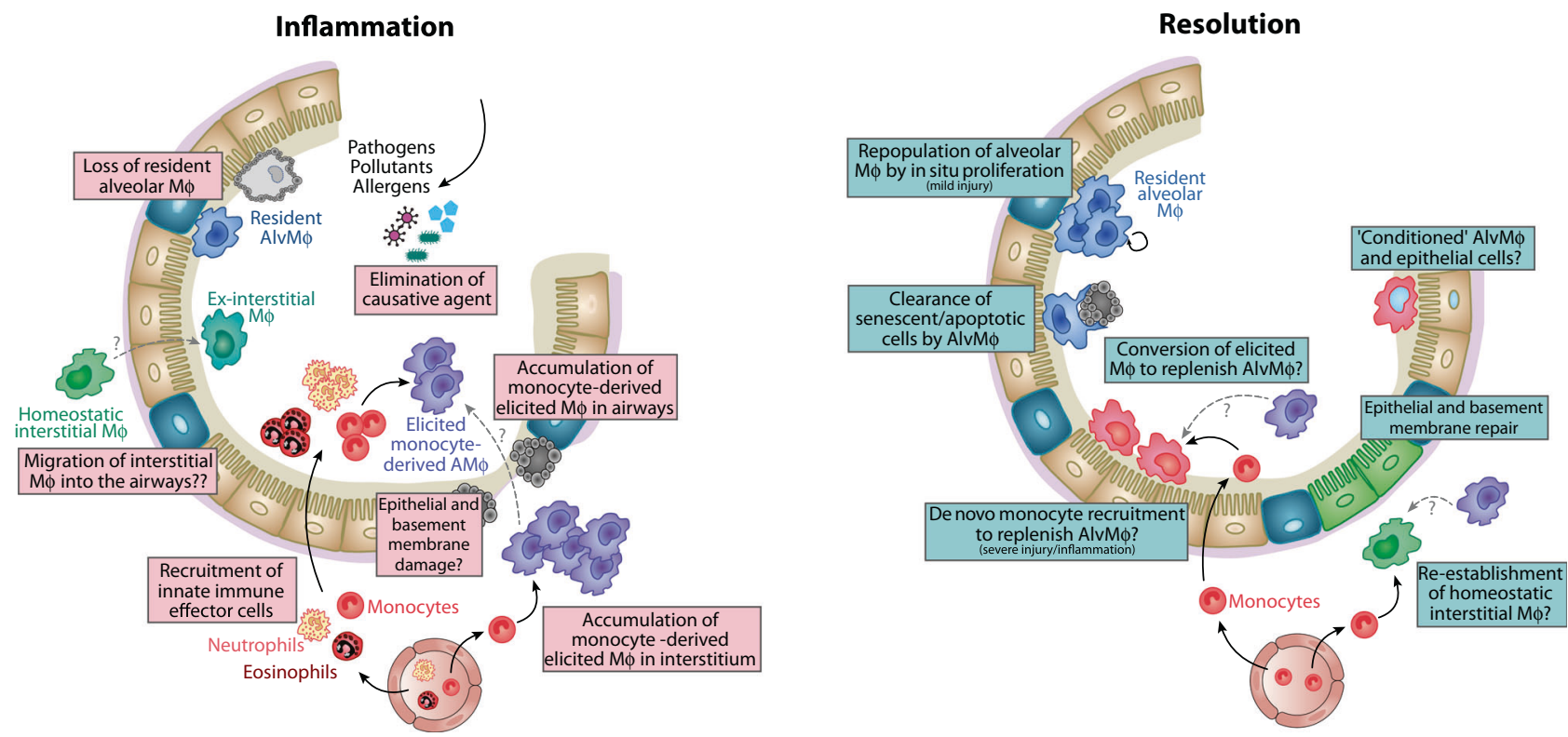

Fig. 3 Pulmonary macrophage dynamics during inflammation and resolution. In inflammation caused by agents such as pathogens, pollutants or allergens, most resident AlvM $\phi s$ are lost and replaced by monocyte-derived $M \phi s$ and perhaps ex-IntM $\phi s$. This occurs in parallel to accumulation of other inflammatory cells such as neutrophils and eosinophils, recruitment of which to the airways is facilitated by chemokines and disrupted barrier integrity. During resolution of the damage caused by acute inflammation, and/or in the face of chronic lowlevel inflammation, residual AlvM $\phi s$ can self-renew through proliferation, clear up dying or dysfunctional cells in the airways, as well as be replenished through conversion of monocyte-derived macrophages and ex-IntM $\phi s$ which are transcriptionally, epigenetically and functionally conditioned by the airway environment to take on AlvM $\phi$ identity.

can and do proliferate under normal physiological conditions ${ }^{15,68}$. While this appears to be insufficient to maintain these populations, it may be sufficient in the absence of monocytes. It is also important to appreciate that self-renewal and derivation from monocytes are not mutually exclusive mechanisms of macrophage maintenance and, in certain contexts, monocyte-derived macrophages proliferate more readily than their embryonic counterparts ${ }^{73,86,87}$. Replenishment by monocytes has been proposed to arise in response to niche availability ${ }^{88}$, although the factors that govern 'availability' remain poorly understood and it is unclear if this differs in distinct subanatomical niches within the lung. Alternatively, there is evidence that different precursors have distinct metabolic states that may determine their long-term persistence in the lung, suggesting that cell-intrinsic properties may also govern replenishment kinetics ${ }^{89}$.

\section{Macrophage origin following injury, inflammation or infections}

The mechanisms that govern macrophage expansion or repopulation following injury, infection or inflammation appear to be dependent on the nature of the insult. Acute inflammation or severe infection often leads to loss of tissue resident macrophages, a phenomenon described as the 'macrophage disappearance reaction' (Fig. 3). For instance, sterile inflammation induced by instillation of LPS in mice leads to a transient loss of AlvM $\phi s$ and expansion of IntM $\phi s$ in the tissue (unpublished observations). IntM $\phi$ expansion is impeded by $\mathrm{C} C \mathrm{r} 2$ deficiency ${ }^{29}$, suggesting a major role for monocyte recruitment in this process. In contrast, AlvM $\phi$ repopulation during inflammation resolution appears to rely exclusively on local proliferation ${ }^{71}$. In contrast, following a more substantial inflammatory insult, such as that induced by administration of bleomycin or silica to model lung fibrosis or infection with influenza, leads to replacement of resident AlvM $\phi s$ with monocyte-derived cells ${ }^{90,91}$. Consistent with this, monocyte-derived cells come to dominate the AM $\phi$ compartment in individuals with severe Sars-CoV-2 infection ${ }^{5}$. Whether this reflects direct effects of severe inflammation on the self-renewal capacity of AlvMфs, or if severe inflammation leads to structural alterations, such as breakdown of basement membrane and epithelial integrity, is unknown. While classical monocytes can enter the airways in response to injury or infection, there is evidence that monocyte-derived, elicited macrophages in the interstitium may also transition to the airways. Whether these alternative differentiation routes influence the fate and function of these cells is currently unclear.

\section{ENVIRONMENTAL IMPRINTING OF LUNG MACROPHAGES}

The diversity of macrophages within the lung results from their plasticity and ability to respond to local environmental cues. In this regard, the pulmonary environment is unusual, even in comparison to other barrier sites, in terms of the wide range of environmental features it presents that can influence immune cell recruitment, activation and function, and that will have a particular impact on lumen-dwelling AlvMфs. These include the unique makeup of the airway fluids (predominantly composed of surfactant and mucins), commensals (bacteria, viruses and fungi) and nutrient levels (e.g., both host and microbial derived metabolites), all of which can change markedly during inflammation.

As well as acting as a lubricant, pulmonary surfactant constituents can also influence AlvM $\phi$ behaviour. For instance, in the absence of surfactant protein D (SP-D) AlvMфs display an unusual $C D 11 b^{\text {hi }}$ phenotype and constitutively produce TNFa ${ }^{92}$. While phenotypic alterations are not evident in AlvM $\phi s$ from naïve SP-A deficient mice ${ }^{93}$, SP-A may amplify IL-4Ra-mediated AlvM $\phi$ activation while regulating their responsiveness to exogenous stimulation through direct interactions with Toll-like receptor 4 (TLR4) and MD-2 ${ }^{93-95}$. As collectins, surfactant conformation can dictate their function in a context-dependent manner, with structural changes altering their binding to targets and in doing so conferring pro- vs anti-inflammatory ability ${ }^{96}$.

The other major constituent of pulmonary fluids is mucus, as vital for lubricating the airways as surfactant, and also for entrapment 
and cilliary clearance of inhaled particles and microbes ${ }^{97}$. Additionally, mucus can be important for control of bacterial infection, regulation of hydration, resolution of inflammation, and modulation of immune and epithelial cell function ${ }^{98,99}$. The main mucins expressed in the lung are Muc5b and Muc5ac, with Muc5b being essential for maintenance of healthy airways ${ }^{97,99}$, while Muc5ac is up-regulated during inflammation ${ }^{100-102}$. Each of these mucins has different properties that determine their dominant function. Mucus also provides a home for commensal lung microbes ${ }^{97}$, which can exert a dramatic effect over airway function, for example through invasion/colonisation, consumption of nutrients, and production of metabolites - all of which can influence pulmonary inflammation and macrophage activation and function. Although most is currently known about bacteria and their products in this context, more widespread metagenomic approaches will increase our understanding of the abundance and diversity of bacterial, viral and fungal commensals in the lung, and how this changes during inflammation and disease.

In terms of nutrients, the airways present AlvMфs with one of the lowest glucose environments in the body, a tissue adaptation that has likely evolved to prevent outgrowth of glucose-hungry opportunistic bacteria ${ }^{103,104}$. Indeed, epithelial cells lining the lung express high levels of glucose transporters apically ${ }^{105}$, with which they can rapidly and efficiently reduce glucose levels in the airways, with the blood and tissues underlying the epithelium possessing over twelve times the levels of glucose than are found in the airway fluids ${ }^{103,105}$. This low-glucose environment, coupled with abundant lipid-rich surfactant, likely plays a central role in governing AlvM $\phi$ metabolism and function. Indeed, it is now clear that metabolism is a central factor in governing macrophage activation and function, with the general principle being that glycolysis may be more associated with 'type 1 ' macrophages, while lipid metabolism tends to be more typical of 'type 2' macrophages (reviewed by ${ }^{106,107}$ ). However, the majority of the work that has established this paradigm has relied on in vitro assessment of bone marrow or monocyte-derived macrophages, with much less understanding of how tissue environments influence macrophage metabolism in vivo. In the context of the lung, we and others have shown that AlvM $\phi$ s display a distinctive metabolic profile, expressing elevated levels of genes associated with lipid metabolism and peroxisome proliferator-activated receptor gamma (PPARY), and reduced expression of genes associated with glycolysis ${ }^{15,20}$. Further, we have shown that glycolysis is a key determinant of AlvM $\phi$ activation and function in type 2 inflammation ${ }^{15}$, suggesting that glycolytic ability, and availability of glucose, may be centrally involved in enabling AlvM $\phi$ activation and function in the airways.

During inflammation, with compromised epithelial integrity, the balance of airway nutrients can change markedly, with reports of elevated airway glucose evident in a range of disease settings, including COPD, cystic fibrosis and asthma ${ }^{108-111}$. This may be particularly relevant in chronic conditions where metabolite balance in the airways can be modified long-term, with consequent longterm alteration of AlvM $\phi$ metabolism and function. In more acute settings, such mechanisms may enable a window of opportunity for glycolytic 'boosting' of AlvM $\phi$ function that will reduce as the epithelium heals and metabolite levels recalibrate, in essence providing a metabolic rheostat for fine-tuning of AlvM $\phi$ activation and function directly linked to how local substrate levels change in line with levels of tissue damage vs resolution.

In contrast to AlvMфs, less is currently known about metabolic control of IntM $\phi$ activation and function. IntM $\phi$ s residing in the more nutrient-rich environment of the lung tissue appear much more glycolytically active than their AlvM $\phi$ counterparts, and are consequently more effective at producing the reactive oxygen species necessary for killing intracellular bacteria such as Mycobacterium tuberculosis ${ }^{20,112}$. In this way, IntM $\phi$ s may be less susceptible to substrate-related functional control than AlvM $\phi s$, though this likely changes if IntMфs migrate into the airways in the context of inflammation as they adapt to this new environment. Indeed, a key outstanding question is to what extent does the metabolic status of monocyte-derived macrophages play in their differentiation to AlvM $\phi s$ during and following an inflammatory insult. It is important, however, to remember that the methods to extract tissue macrophages can have profound effects on their biology, including their transcriptional and metabolic status. Given that IntM $\phi$ s are embedded in the tissue, it is plausible that some of the differences seen in their metabolic profile may reflect their response to extraction.

As described above, there is continual bi-directional crosstalk between macrophages and the structural/stromal cells that comprise their niche, and this crosstalk leads to niche-specific outcomes for macrophage recruitment, differentiation and function. Removing macrophages from their niche leads to phenotypic and transcriptional changes, directly demonstrating the need for continual crosstalk with structural cells ${ }^{3}$. In the airways, AlvM $\phi s$ are highly dependent on GM-CSF for their development and maintenance ${ }^{78,113-117}$, consistent with their high expression of GM-CSF receptor. Consequently, disruption to the GM-CSF-GMCSFR axis leads to defective AlvM $\phi$ differentiation and the development of PAP in both mice and humans ${ }^{45-50}$. Recent work using GM-CSF reporter mice and cell-specific deletion of GM-CSF has identified alveolar type 2 epithelial cells as the indispensable source of GM-CSF during the pre- and post-natal stages of AlvM $\phi$ development from foetal progenitors ${ }^{117}$. Interestingly, although innate lymphoid cells (ILCS) are major sources of GM-CSF in the steady state lung, haematopoietic deletion of GM-CSF does not affect AlvM $\phi$ development ${ }^{117}$. Moreover, although it has been proposed that GM-CSF may control AlvMфs indirectly through induction of $1 / 6, \| 113$ and Csf1 expression in lung basophils ${ }^{118}$, genetic depletion of basophils has little, if any, effect on AlvM $\phi$ number or phenotype ${ }^{117}$. Indeed, that CD11c-mediated deletion of Csf2rb, which encodes one of the GM-CSFR subunits, or Stat5, which lies downstream of GM-CSFR, leads to aborted differentiation of AlvM $\phi s^{119,120}$ supports the notion that GM-CSF acts directly on developing AlvM $\phi s$.

As mentioned above, crosstalk between AlvM $\phi s$ and alveolar epithelial cells also involves the TGF $\beta$-TGF $\beta$ R axis. TGF $\beta$ is a potent immunomodulatory cytokine which is abundant in the mouse and human lung in health. It is produced in a latent form and must be converted to active TGF $\beta$ to have biological effects. In the airways, integrin-mediated activation is thought to be the principal mechanism of TGF $\beta$ activation. Specifically, the av $\beta 6$ integrin, which is expressed by alveolar epithelial cells, is crucial for generating active TGF ${ }^{121}$. Consequently, genetic disruption of Itgb6, which encodes integrin $\beta 6$, leads to development of emphysema due to excessive production of MMP12 by dysfunctional AlvM $\phi s ;$ a phenotype that can be rescued by constitutive expression of TGF $\beta 1^{121,122}$. Human AM $\phi$ s are known to have a gene signature consistent with TGF $\beta$ R signalling ${ }^{123}$ and myeloidspecific deletion of TGF $\beta R$ in mice leads to aborted AlvM $\phi$ development, demonstrating a need for cell intrinsic TGF $\beta$ R for this process ${ }^{124,125}$. Interestingly, although many cells can produce TGF $\beta$, macrophages themselves are thought to be an important source ${ }^{123,124}$. Moreover, AlvM $\phi s$ can facilitate integrin-mediated release of active TGF $\beta$ through production of amphiregulin, at least in the context of helminth infection ${ }^{126}$. Loss of TGF $\beta R$ signalling leads to reduced expression of GM-CSFR, suggesting cooperation between these factors ${ }^{124}$. However, the indispensable nature of TGF $\beta$ in their development makes assessing its role in regulation of AlvM $\phi$ behaviour during homeostasis difficult. Nevertheless, TGF $\beta$ is thought to upregulate the inhibitory receptor CD200R1, which is known to be key for maintaining the activation threshold of AlvM $\phi s^{127}$. Consistent with this, loss of autocrine TGF $\beta$ leads to spontaneous production of proinflammatory cytokines and chemokines by AlvM $\phi s^{123}$. 
Both GM-CSF and TGF $\beta$ induce expression of the transcription factor PPARy, which is considered the master transcription factor for AlvM $\phi s^{43,124}$. Mice with myeloid-specific deletion of Pparg also develop PAP, consistent with regulation of molecules involved in lipid catabolism by PPAR ${ }^{43,128,129}$. Given that PPARY is expressed by macrophages in other tissues, including splenic red pulp macrophages and macrophages of the erythroblastic islands in the bone marrow ${ }^{129,130}$, until recently, it remained unclear how specificity was conferred to AlvMфs. We recently uncovered the transcription factor early growth response 2 (EGR2) as a key evolutionarily conserved regulator of AlvM $\phi$ differentiation downstream of PPARy in the lung but not spleen ${ }^{125}$. Interestingly, mice with Egr2 deficient AlvM $\phi$ s and individuals with mutations in EGR2 do not appear to develop spontaneous PAP ${ }^{125,131}$, demonstrating that PPAR $\gamma$ must cooperate with other transcriptional regulators to regulate distinct aspects of AlvM $\phi$ biology. For instance, EGR2 appears particularly important for regulating expression of adhesion molecules, chemotactic machinery and apparatus for the detection and elimination of respiratory pathogens ${ }^{125}$. EGR2 appears to maintain expression of CCAAT/enhancer-binding protein beta (C/EBP $\beta)$, which has been implicated in AlvM $\phi$ differentiation ${ }^{132}$. The transcription factors Bhlhe40 and Bhlhe41 have also been shown to control the phenotypic identity and proliferative capacity of AlvM $\phi s$, and seem to rely on TGF $\beta R$ signalling in a PPAR $\gamma$-independent manner ${ }^{133}$. The histone deacetylase (HDAC) sirtuin 1 (SIRT1) also plays a key role in regulating the proliferative activity of AlvM $\phi s^{134}$. Finally, Bach2 (B lymphoid transcriptional repressor BTB and CNC homology 2) has been shown to be essential for surfactant regulation by AlvM $\phi s^{135}$. Thus, while much progress has been made in understanding the transcriptional control of lung macrophages, if and how these transcriptional regulators interact or cooperate to control the discrete molecular programmes required for homeostatic function of AlvMфs is only starting to be understood and warrants further study using state-of-the-art technologies.

The environmental control of IntM $\phi s$ is much less well understood. Despite high expression of CX3CR1 by at least some IntM $\phi s$, their survival, phenotype and proliferative capacity is unaffected by $\mathrm{C} \times 3 \mathrm{Cr} 1$ deficiency ${ }^{14}$. Unlike their alveolar counterparts, IntM $\phi s$ rely on signalling through CSF1R for their development and maintenance as evidenced by their depletion with antiCSF1R antibody treatment ${ }^{28}$ and failure to develop from $\mathrm{Csflr}^{-1-}$ precursors in a competitive bone marrow chimera setting ${ }^{136}$. The relative role of the ligands for the CSF1R, M-CSF and IL-34, has not been examined exhaustively. For instance, although $\mathrm{CDC} 2 \mathrm{~s}$ are reported to be affected in I/34 $4^{\text {LacZ/LacZ }}$ mice ${ }^{137}$, these cells were simply defined as $C D 11 c^{+} C D 11 b^{+}$non-AlvM $\phi s$ and it is highly likely this compartment contains both IntM $\phi s$ and $\mathrm{CDC} 2 \mathrm{~s}$. Similarly, analysis of $C s f 1^{\text {op/op }}$ mice, which have a naturally occurring inactivating mutation in the Csf1 gene, has shown an effect on the abundance of $\mathrm{CD}_{169^{+}}$but not CD169 $\operatorname{IntM} \phi s^{28}$, suggesting differential reliance on M-CSF by discrete IntM $\phi$ subsets. Application of novel reporter and conditional "KO" mice, such as those used to identify the cellular sources of M-CSF in the lymph node and spleen ${ }^{138,139}$, should help discern the relative roles and cellular origin of M-CSF and IL-34 in regulating survival and differentiation of IntM $\phi$ subsets.

The downstream molecular pathways that govern $\operatorname{Int} M \phi$ differentiation remain largely elusive. Although expression of Maf, Mafb, Irf5, Jun and Atf3 have been identified through scRNAseq studies as highly expressed by murine $\ln \mathrm{t} M \phi \mathrm{s}^{6,125}$, if and how these transcription factors control their differentiation remains unexplored. Furthermore, given that some of these (e.g., IRF5) have been implicated in AlvM $\phi$ homeostasis ${ }^{140}$, high expression does not always equate to specificity. Importantly, although dispensable for the phenotypic identity and survival of IntMфs, $\beta$ catenin signalling has recently been implicated in the control of the metabolic profile of IntM $\phi s$, in response to the Wnt family molecule Rspondin3 derived from pulmonary endothelial cells ${ }^{141}$ IntMфs are intimately associated with extracellular matrix and interaction with collagen via the collagen receptor, LAIR1, appears to alter the composition of the IntM $\phi$ pool. Notably, LAIR1 appears to regulate CSF1R expression ${ }^{142}$ and therefore interactions with the ECM may regulate macrophage longevity, although this remains to be tested experimentally.

Thus, it is clear that the lung environment exerts multiple layers of control over macrophage development, activation and function in health and during inflammation. This highlights the current over-reliance on in vitro methods to research lung macrophages, particularly for human research, which are likely of questionable relevance. Innovative new approaches are needed that better reflect the lung environment, such as 'lung on a chip'143-146 $\operatorname{organoids}^{147,148}$, and maintenance of whole lung tissue ex vivo.

\section{MACROPHAGES IN PULMONARY FIBROSIS}

Despite their key roles in lung homeostasis, macrophages are implicated in the pathogenesis of many chronic lung pathologies, including pulmonary fibrosis (PF). PF is a common feature of a group of conditions known as interstitial lung diseases (ILDs), where excessive ECM deposition leads to irreversible scarring of the lung (reviewed by ${ }^{149,150}$ ). In many cases the cause of pulmonary fibrosis is not identified (idiopathic pulmonary fibrosis (IPF)), whereas in others it can be attributed to exposure certain occupational substances (e.g., asbestos, silica) or drugs (e.g., bleomycin, methotrexate). Moreover, there are indications that following severe coronavirus disease 19 (COVID-19), certain individuals develop pulmonary fibrosis ${ }^{151}$, although whether this results in permanent, irreversible scarring is still being understood.

While the prevailing school of thought is that PF arises from ineffective repair of airway epithelium following repetitive injury, there is now compelling evidence that macrophages contribute to PF pathology ${ }^{152}$ and that targeting macrophages could be beneficial in human disease ${ }^{153}$. First, there is vast macrophage accumulation in the lung parenchyma during PF and experimental fibrosis where they co-localise with collagen-producing myofibroblasts and support their proliferation and function through production of PDGFa, PDGF $\beta$, TGF $\beta 1$ and Galectin-3 $30,154-157$ Macrophages have also been shown to be rich sources of osteopontin (encoded by Spp1/SPP1) 32,90,155,158, which has long been established as a pro-fibrotic mediator, in part through activation of TGF $\beta 1^{158}$. Osteopontin-producing macrophages are found in the airways and parenchyma of IPF individuals ${ }^{159}$, although highest expression is attributed to $M A F B^{+} P P A R G^{-}$ macrophages, which most likely represent IntM $\phi s^{32}$. Moreover, high expression of inhibitors of collagenolytic enzymes, including tissue inhibitor of metalloproteinases 1 (TIMP1) and TIMP2 is a feature of fibrosis-associated macrophages ${ }^{154}$. Studies in mice have shown macrophages to be able to produce certain collagens (e.g., collagen VI), and collagen VI deficiency limited to the haematopoietic compartment ameliorates experimental bleomycin-induced fibrosis ${ }^{160}$. Interestingly, however, elevated expression of matrix metalloproteinases, such as MMP-9, MMP-12 and MMP-14, also defines fibrosis-associated macrophages across species. Thus, the relative contribution of collagen production versus collagen clearance remains poorly understood.

Macrophage accumulation results, at least in part, from de novo recruitment of $\mathrm{CCR}^{+}$monocytes in both experimental models and human $\mathrm{PF}^{161}$. The presence of $\mathrm{CCR}^{+}$monocytes and their macrophage progeny correlates with the presence of fibrotic tissue in mouse and $\operatorname{man}^{161}$, and bleomycin-induced experimental fibrosis can be exacerbated by adoptive transfer of classical monocytes ${ }^{162}$. Consistent with this, experimental fibrosis is blunted in monocytopenic CCr2 deficient mice ${ }^{161,163,164}$, by neutralisation of the CSF1-CSF1R axis ${ }^{154}$ or by rendering monocyte-derived macrophages susceptible to apoptosis ${ }^{165}$. 
Moreover, one of the few treatments for PF, pirfenidone, has recently been shown to reduce accumulation of $\mathrm{CCR}^{+}$monocytes in bleomycin-induced experimental fibrosis ${ }^{161}$. However, the Akira group has suggested that developmentally distinct, profibrotic monocytes termed "segregated-nucleus-containing atypical monocytes" (SatM) arise in the context of experimental lung fibrosis and are responsible for driving disease ${ }^{166}$. So-called 'SatM' appear to depend on the transcription factor C/EBP $\beta$, but derive from $\mathrm{FcER}^{+}$granulocyte/macrophage progenitors (GMPs) and not macrophage/DC progenitors (MDPs) ${ }^{166}$. Indeed, work since has described distinct pathways to generate monocytes from GMPs and MDPs in health and following infection ${ }^{167}$. How these SatM relate to the CCR2-dependent monocytes described in other studies remains unclear.

Whether pro-fibrotic macrophages are limited to the lung parenchyma or if monocyte-derived $A M \phi s$ also contribute to fibrosis is still under debate $e^{90,125,154,156}$. In support of the latter, sustained epithelial injury is a feature of human $\mathrm{PF}^{155,168}$ and reducing epithelial damage through administration of a specific inhibitor of sphingosine kinase 1, which is elevated in IPF lungs, reduces experimental fibrosis, at least in part by reducing recruitment of fibrogenic monocytes ${ }^{169}$. Attributing key pathogenic roles to macrophage subsets is made difficult by the breakdown in clear phenotypic boundaries between parenchymal and AlvM $\phi s$ in the context of inflammation and fibrosis, and by the fact that, at least some, IntM $\phi$ s may differentiate into AlvMфs during lung repair ${ }^{125,156}$. PF incidence and severity positively correlates with age ${ }^{170-172}$ and given monocytes may progressive replace embryonically-derived AlvM $\phi s$ with age, it is intriguing to speculate that these two phenomena could be related. In addition to blood-derived monocytes, it is plausible that macrophages in the pleural cavity may contribute to the pro-fibrotic pool of macrophages in the lung. Consistent with this notion, fibrosis in IPF patients is often concentrated in the subpleural region ${ }^{150}$ and there is transcriptional similarity between pleural and $\mathrm{MHCll}^{-}$ IntMфs in mice 27,173 . Moreover, it's been suggested serous cavity macrophages may contribute to tissue repair in neighbouring solid organs following injury in mice ${ }^{174,175}$. However, elegant intersectional genetics and a combination of injury models have shown that, while pleural GATA6+ macrophages may accumulate on the pleural membrane, they do not migrate deep into the lung parenchyma nor are they essential for fibrogenesis or resolution $^{176}$.

Why macrophages become excessively pro-fibrotic is only starting to be understood, although, again, this probably reflects their plasticity. IL-4, IL-13, IL-33 and TGF $\beta$ have all been implicated in altering macrophage behaviour in PF (reviewed by ${ }^{152}$ ), but the relative and combinatorial roles of these factors is poorly understood. Recently, overactive Notch signalling has been implicated in the pro-fibrotic behaviour of macrophages, as deletion of RBPJ reduces fibrosis in mice through abrogating TGF $\beta$ production by $\mathrm{Ly}^{6 \mathrm{C}^{\mathrm{hi}} \mathrm{MHClI}}{ }^{+}$monocyte-derived macrophages ${ }^{177}$. Whether these effects are attributable to airway or IntM $\phi s$ is difficult to discern in this study ${ }^{177}$. TGF $\beta$ is of particular interest given its long-standing role in tissue fibrosis ${ }^{178}$. Recent work has uncovered discrete functions of TGF $\beta$ isoforms in the fibrotic process $^{179}$. However, if and how excessive TGF $\beta$ isoforms influence macrophage function in the fibrotic niche in vivo has not been tested directly.

Like in many pathologies, the role of macrophages in lung fibrosis is very much context dependent. The self-resolving nature of some experimental models allows macrophage dynamics and behaviour to be assessed during fibrosis regression and resolution, something that cannot be gleaned from human disease. This has revealed that severe lung injury leads to almost complete replacement of embryonically derived AlvMфs with monocytederived cells ${ }^{90,125}$. We have recently shown that this process is highly dependent on the transcription factor EGR2 and that
EGR2-dependent monocyte-derived AM $\phi$ s are indispensable for resolution of fibrosis and restoration of airway homeostasis ${ }^{125}$, findings consistent with older non-specific depletion studies in mice ${ }^{162}$. There is evidence this may involve direct clearance of collagen by (monocyte-derived) AM $\phi$ s. For instance, genetic ablation of milk fate globule epidermal growth factor 8 (Mfge8), a receptor typically associated with efferocytosis, leads to failed collagen clearance following bleomycin-induced injury ${ }^{180}$. Moreover, macrophage-derived ApoE may facilitate binding and targeting of type I collagen for phagocytosis via the low-density lipoprotein receptor-related protein 1 (LRP1) ${ }^{181}$. Pro-resolution roles of monocyte-derived AM $\phi$ s are also seen following influenza infection and their absence can result in the development of fibrosis, at least in mice ${ }^{182}$. Such functions may include metabolic rewiring of monocyte-derived macrophages as deletion of Acod1, the enzyme required for generation of the metabolite itaconate, leads to persistent fibrosis ${ }^{183}$. Thus, while generally considered as key pro-fibrotic cellular players, monocyte-derived AM $\phi s$ appear to have a crucial role in re-establishing lung homeostasis and may hold great promise for therapeutic targeting to promote fibrosis regression and lung repair.

\section{CONCLUSION}

The past few years have seen a leap forwards in our understanding of pulmonary macrophage development, heterogeneity and function, and how environmental features of the lung can exert a dramatic influence over these processes in both disease and in health. A major challenge for the coming years is to develop much greater clarity on how different inflammatory conditions alter pulmonary macrophage subset diversity and function, in particular in the context of human disease, to identify core mechanisms that might enable development of the targeted therapeutics of the future.

\section{REFERENCES}

1. Mills, C. D., Kincaid, K., Alt, J. M., Heilman, M. J. \& Hill, A. M. M-1/M-2 macrophages and the Th1/Th2 paradigm. J. Immunol. 164, 6166-6173 (2000).

2. Murray, P. J. et al. Macrophage activation and polarization: nomenclature and experimental guidelines. Immunity 41, 14-20 (2014).

3. Lavin, Y. et al. Tissue-resident macrophage enhancer landscapes are shaped by the local microenvironment. Cell 159, 1312-1326 (2014).

4. Mulder, K. et al. Cross-tissue single-cell landscape of human monocytes and macrophages in health and disease. Immunity 54, 1883-1900.e5 (2021).

5. Liao, M. et al. Single-cell landscape of bronchoalveolar immune cells in patients with COVID-19. Nat. Med. 26, 842-844 (2020).

6. Sajti, E. et al. Transcriptomic and epigenetic mechanisms underlying myeloid diversity in the lung. Nat. Immunol. 21, 221-231 (2020).

7. Leach, S. M. et al. Human and mouse transcriptome profiling identifies crossspecies homology in pulmonary and lymph node mononuclear phagocytes. Cell Rep. 33, 108337 (2020).

8. Lavin, Y. et al. Innate immune landscape in early lung adenocarcinoma by paired single-cell analyses. Cell 169, 750-765.e17 (2017).

9. Zilionis, R. et al. Single-cell transcriptomics of human and mouse lung cancers reveals conserved myeloid populations across individuals and species. Immunity 50, 1317-1334.e10 (2019).

10. Raredon, M. S. B. et al. Single-cell connectomic analysis of adult mammalian lungs. Sci. Adv. 5, eaaw3851 (2019).

11. Travaglini, K. J. et al. A molecular cell atlas of the human lung from single-cell RNA sequencing. Nature 587, 619-625 (2020).

12. Gautier, E. L. et al. Gene-expression profiles and transcriptional regulatory pathways that underlie the identity and diversity of mouse tissue macrophages. Nat. Immunol. 13, 1118-1128 (2012).

13. Bharat, A. et al. Flow cytometry reveals similarities between lung macrophages in humans and mice. Am. J. Respir. Cell Mol. Biol. 54, 147-149 (2016).

14. Gibbings, S. L. et al. Three unique interstitial macrophages in the murine lung at steady state. Am. J. Respir. Cell Mol. Biol. 57, 66-76 (2017).

15. Svedberg, F. R. et al. The lung environment controls alveolar macrophage metabolism and responsiveness in type 2 inflammation. Nat. Immunol. 20, 571-580 (2019). 
16. Becher, B. et al. High-dimensional analysis of the murine myeloid cell system. Nat. Immunol. 15, 1181-1189 (2014).

17. Hoffmann, F. M. et al. Distribution and interaction of murine pulmonary phagocytes in the naive and allergic lung. Front Immunol. 9, 1046 (2018).

18. Schyns, J. et al. Non-classical tissue monocytes and two functionally distinct populations of interstitial macrophages populate the mouse lung. Nat. Commun. 10, 3964 (2019).

19. Hume, P. S. et al. Localization of macrophages in the human lung via designbased stereology. Am J. Respir. Crit. Care Med. 201, 1209-1217 (2020).

20. Huang, L., Nazarova, E. V., Tan, S., Liu, Y. \& Russell, D. G. Growth of mycobacterium tuberculosis in vivo segregates with host macrophage metabolism and ontogeny. J. Exp. Med. 215, 1135-1152 (2018).

21. Bosteels, C. et al. Inflammatory type 2 cDCs acquire features of CDC1s and macrophages to orchestrate immunity to respiratory virus infection. Immunity 52, 1039-1056.e9 (2020).

22. Ono, Y. et al. CD11c+M1-like macrophages (MФs) but not CD206+ M2-like MФ are involved in folliculogenesis in mice ovary. Sci. Rep. 8, 8171-10 (2018).

23. Rőszer, $\mathrm{T}$. Understanding the mysterious $\mathrm{M} 2$ macrophage through activation markers and effector mechanisms. Mediators Inflamm. 2015, 816460 (2015).

24. Saini, Y. et al. Gene expression in whole lung and pulmonary macrophages reflects the dynamic pathology associated with airway surface dehydration. BMC Genomics 15, 726 (2014).

25. Lai, J. F. et al. Critical role of macrophages and their activation via MyD88-NFKB signaling in lung innate immunity to Mycoplasma pneumoniae. PLoS One 5, e14417 (2010).

26. Nahrendorf, M. \& Swirski, F. K. Abandoning M1/M2 for a network model of macrophage function. Circ. Res. 119, 414-417 (2016).

27. Chakarov, S. et al. Two distinct interstitialmacrophage populations coexist across tissues in specific subtissular niches. Science 363, eaau0964 (2019).

28. Ural, B. B. et al. Identification of a nerve-associated, lung-resident interstitial macrophage subset with distinct localization and immunoregulatory properties. Sci. Immunol. 5, eaax8756 (2020)

29. Sabatel, C. et al. Exposure to bacterial CpG DNA protects from airway allergic inflammation by expanding regulatory lung interstitial macrophages. Immunity 46, 457-473 (2017).

30. Yu, Y.-R. A. et al. Flow cytometric analysis of myeloid cells in human blood, bronchoalveolar lavage, and lung tissues. Am. J. Respir. Cell Mol. Biol. 54, 13-24 (2016).

31. McQuattie-Pimentel, A. C. et al. The lung microenvironment shapes a dysfunctional response of alveolar macrophages in aging. J. Clin. Invest. 131, e140299 (2021).

32. Morse, C. et al. Proliferating SPP1/MERTK-expressing macrophages in idiopathic pulmonary fibrosis. Eur. Respir. J. 54, 1802441 (2019).

33. Vieira Braga, F. A. et al. A cellular census of human lungs identifies novel cell states in health and in asthma. Nat. Med. 25, 1153-1163 (2019).

34. Mould, K. J. et al. Airspace macrophages and monocytes exist in transcriptionally distinct subsets in healthy adults. Am J. Respir. Crit. Care Med. 203, 946-956 (2021).

35. Evren, E. et al. Distinct developmental pathways from blood monocytes generate human lung macrophage diversity. Immunity 54, 259-275.e7 (2021).

36. Aberdein, J. D., Cole, J., Bewley, M. A., Marriott, H. M. \& Dockrell, D. H. Alveolar macrophages in pulmonary host defence the unrecognized role of apoptosis as a mechanism of intracellular bacterial killing. Clin. Exp. Immunol. 174, 193-202 (2013).

37. Preston, J. A. et al. Alveolar macrophage apoptosis-associated bacterial killing helps prevent murine pneumonia. Am. J. Respir. Crit. Care Med. 200, 84-97 (2019).

38. Schneider, C. et al. Alveolar macrophages are essential for protection from respiratory failure and associated morbidity following influenza virus infection. PLoS Pathog. 10, e1004053 (2014).

39. $\mathrm{Xu}, \mathrm{J}$. et al. Scavenger receptor MARCO orchestrates early defenses and contributes to fungal containment during cryptococcal infection. J. Immunol. 198, 3548-3557 (2017).

40. Dorrington, M. G. et al. MARCO is required for TLR2- and Nod2-mediated responses to Streptococcus pneumoniae and clearance of pneumococcal colonization in the murine nasopharynx. J. Immunol. 190, 250-258 (2013).

41. Arredouani, M. et al. The scavenger receptor MARCO is required for lung defense against pneumococcal pneumonia and inhaled particles. J. Exp. Med. 200, 267-272 (2004).

42. Hussell, T. \& Bell, T. J. Alveolar macrophages: plasticity in a tissue-specific context. Nat. Rev. 14, 81-93 (2014).

43. Schneider, C. et al. Induction of the nuclear receptor PPAR- $\gamma$ by the cytokine GM-CSF is critical for the differentiation of fetal monocytes into alveolar macrophages. Nat. Immunol. 15, 1026-1037 (2014).
44. Gibbings, S. L. et al. Transcriptome analysis highlights the conserved difference between embryonic and postnatal-derived alveolar macrophages. Blood 126 1357-1366 (2015).

45. Nishinakamura, R. et al. Mice deficient for the IL-3/GM-CSF/IL-5 beta c receptor exhibit lung pathology and impaired immune response, while beta IL3 receptordeficient mice are normal. Immunity 2, 211-222 (1995).

46. Robb, L. et al. Hematopoietic and lung abnormalities in mice with a null mutation of the common beta subunit of the receptors for granulocytemacrophage colony-stimulating factor and interleukins 3 and 5. Proc. Natl Acad. Sci. U.S.A. 92, 9565-9569 (1995).

47. Kitamura, $\mathrm{T}$. et al. Idiopathic pulmonary alveolar proteinosis as an autoimmune disease with neutralizing antibody against granulocyte/macrophage colonystimulating factor. J. Exp. Med. 190, 875-880 (1999).

48. Suzuki, T. et al. Familial pulmonary alveolar proteinosis caused by mutations in CSF2RA. J. Exp. Med. 205, 2703-2710 (2008).

49. van de Laar, L. et al. Yolk sac macrophages, fetal liver, and adult monocytes can colonize an empty niche and develop into functional tissue-resident macrophages. Immunity https://doi.org/10.1016/j.immuni.2016.02.017 (2016).

50. Stanley E. et al. Granulocyte/macrophage colony-stimulating factor-deficient mice show no major perturbation of hematopoiesis but develop a characteristic pulmonary pathology. Proc Natl Acad Sci USA. 91, (1994).

51. Peters, D. M. et al. TGF- $\beta$ directs trafficking of the epithelial sodium channel $\mathrm{ENaC}$ which has implications for ion and fluid transport in acute lung injury. Proc. Natl Acad. Sci. U.S.A. 111, E374-E383 (2014).

52. Han, S. W. \& Roman, J. Fibronectin induces cell proliferation and inhibits apoptosis in human bronchial epithelial cells: pro-oncogenic effects mediated by PI3-kinase and NF-kappa B. Oncogene 25, 4341-4349 (2006).

53. Bourdonnay, E. et al. Transcellular delivery of vesicular SOCS proteins from macrophages to epithelial cells blunts inflammatory signaling. J. Exp. Med. 212, 729-742 (2015).

54. Draijer, C. et al. Resident alveolar macrophage-derived vesicular SOCS3 dampens allergic airway inflammation. FASEB J. 34, 4718-4731 (2020).

55. Schyns, J., Bureau, F. \& Marichal, T. Lung interstitial macrophages: past, present, and future. J. Immunol. Res 2018, 5160794 (2018).

56. Bedoret, D. et al. Lung interstitial macrophages alter dendritic cell functions to prevent airway allergy in mice. J. Clin. Invest. 119, 3723-3738 (2009).

57. Kawano, $\mathrm{H}$. et al. IL-10-producing lung interstitial macrophages prevent neutrophilic asthma. Int. Immunol. 28, 489-501 (2016).

58. Hoppstädter, J. et al. Differential cell reaction upon toll-like receptor 4 and 9 activation in human alveolar and lung interstitial macrophages. Respir. Res. 11, 124-15 (2010).

59. Plantinga, $M$. et al. Conventional and monocyte-derived $C D 11 \mathrm{~b}(+)$ dendritic cells initiate and maintain T helper 2 cell-mediated immunity to house dust mite allergen. Immunity 38, 322-335 (2013).

60. Ren, J. et al. Intranasal delivery of MSC-derived exosomes attenuates allergic asthma via expanding IL-10 producing lung interstitial macrophages in mice. Int Immunopharmacol. 91, 107288 (2021).

61. Brody, A. et al. Interstitial pulmonary macrophages produce platelet-derived growth factor that stimulates rat lung fibroblast proliferation in vitro. J. Leukoc. Biol. 6, 640-648 (1992).

62. De Schepper, S. et al. Self-maintaining gut macrophages are essential for intestinal homeostasis. Cell 175, 400-415.e13 (2018).

63. van Furth, R. et al. The mononuclear phagocyte system: a new classification of macrophages, monocytes, and their precursor cells. Bull. World Health Organ 46, 845-852 (1972).

64. Schulz, C. et al. A lineage of myeloid cellsindependent of Myb and hematopoietic stem cells. Science 336, 86-90 (2012).

65. Gomez Perdiguero, E. et al. Tissue-resident macrophages originate from yolksac-derived erythro-myeloid progenitors. Nature 518, 547-551 (2015).

66. Hoeffel, G. et al. Adult Langerhans cells derive predominantly from embryonic fetal liver monocytes with a minor contribution of yolk sac-derived macrophages. J. Exp. Med. 209, 1167-1181 (2012).

67. Ginhoux, F. et al. Fate mapping analysisreveals that adult microglia derive from primitive macrophages. Science 330, 841-845 (2010).

68. Hashimoto, D. et al. Tissue-resident macrophages self-maintain locally throughout adult life with minimal contribution from circulating monocytes. Immunity 38, 792-804 (2013).

69. Hoeffel, G. et al. C-Myb(+) erythro-myeloid progenitor-derived fetal monocytes give rise to adult tissue-resident macrophages. Immunity 42, 665-678 (2015).

70. Sheng, J., Ruedl, C. \& Karjalainen, K. Most tissue-resident macrophages except microglia are derived from fetal hematopoietic stem cells. Immunity 43, 382-393 (2015).

71. Liu, Z. et al. Fate mapping via Ms4a3-expression history traces monocytederived. Cells Cell 178, 1509-1525.e19 (2019). 
72. Shaw, T. N. et al. Tissue-resident macrophages in the intestine are long lived and defined by Tim-4 and CD4 expression. J. Exp. Med. 215, 1507-1518 (2018).

73. Bain, C. C. et al. Long-lived self-renewing bone marrow-derived macrophages displace embryo-derived cells to inhabit adult serous cavities. Nat. Commun. 7, ncomms11852 (2016).

74. Epelman, S. et al. Embryonic and adult-derived resident cardiac macrophages are maintained through distinct mechanisms at steady state and during inflammation. Immunity 40, 91-104 (2014).

75. Tarling, J. D., Lin, H. S. \& Hsu, S. Self-renewal of pulmonary alveolar macrophages: evidence from radiation chimera studies. J. Leukoc. Biol. 42, 443-446 (1987).

76. Goldmann, T. et al. A new type of microglia gene targeting shows TAK 1 to be pivotal in CNS autoimmune inflammation. Nat. Neurosci. 16, 1618-1626 (2013).

77. Tan, S. Y. S. \& Krasnow, M. A. Developmental origin of lung macrophage diversity. Development 143, 1318-1327 (2016).

78. Guilliams, M. et al. Alveolar macrophages develop from fetal monocytes that differentiate into long-lived cells in the first week of life via GM-CSF. J. Exp. Med. 210, 1977-1992 (2013)

79. Alenghat, E. \& Esterly, J. R. Alveolar macrophages in perinatal infants. Pediatrics 74, 221-223 (1984)

80. Eguíluz-Gracia, I. et al. Long-term persistence of human donor alveolar macrophages in lung transplant recipients. Thorax https://doi.org/10.1136/thoraxjnl2016-208292 (2016).

81. Nayak, D. K. et al. Long-term persistence of donor alveolar macrophages in human lung transplant recipients that influences donor-specific immune responses. Am. J. Transpl. 16, 2300-2311 (2016).

82. Rongvaux, A. et al. Development and function of human innate immune cells in a humanized mouse model. Nat. Biotechnol. 32, 364-372 (2014).

83. Byrne, A. J. et al. Dynamics of human monocytes and airway macrophages during healthy aging and after transplant. J. Exp. Med. 217, e20191236 (2020).

84. Hawley, C. A. et al. Csf1r-mApple transgene expression and ligand binding in vivo reveal dynamics of CSF1R expression within the mononuclear phagocyte system. J. Immunol. 200, 2209-2223 (2018).

85. Dyer, D. P. et al. Chemokine receptor redundancy and specificity are context dependent. Immunity 50, 378-389.e5 (2019).

86. Jenkins, S. J. et al. IL-4 directly signals tissue-resident macrophages to proliferate beyond homeostatic levels controlled by CSF-1. J. Exp. Med. 210, 2477-2491 (2013).

87. Davies, L. C. et al. Distinct bone marrow-derived and tissue-resident macrophage lineages proliferate at key stages during inflammation. Nat. Commun. 4, 1886 (2013).

88. Guilliams, M. \& Scott, C. L. Does niche competition determine the origin of tissue-resident macrophages? Nat. Rev. 17, 451-460 (2017).

89. Li, F., Okreglicka, K. M., Pohlmeier, L. M., Schneider, C. \& Kopf, M. Fetal monocytes possess increased metabolic capacity and replace primitive macrophages in tissue macrophage development. EMBO J. 39, e103205 (2020).

90. Misharin, A. V. et al. Monocyte-derived alveolar macrophages drive lung fibrosis and persist in the lung over the life span. J. Exp. Med. 214, 2387-2404 (2017).

91. Aegerter, $\mathrm{H}$. et al. Influenza-induced monocyte-derived alveolar macrophages confer prolonged antibacterial protection. Nat. Immunol. 21, 145-157 (2020).

92. Hortobágyi, L. et al. Surfactant protein D inhibits TNF-alpha production by macrophages and dendritic cells in mice. J. Allergy Clin. Immunol. 122, 521-528 (2008).

93. Minutti, C. M. et al. Local amplifiers ofIL-4Ra-mediated macrophage activation promote repair in lung and liver. Science 356, 1076-1080 (2017).

94. Yamada, C. et al. Surfactant protein A directly interacts with TLR4 and MD-2 and regulates inflammatory cellular response. Importance of supratrimeric oligomerization. J. Biol. Chem. 281, 21771-21780 (2006).

95. Sano, $\mathrm{H}$. et al. Pulmonary surfactant protein $\mathrm{A}$ modulates the cellular response to smooth and rough lipopolysaccharides by interaction with CD14. J. Immunol. 163, 387-395 (1999)

96. Murugaiah, V., Tsolaki, A. G. \& Kishore, U. Collectins: innate immune pattern recognition molecules. Adv. Exp. Med. Biol. 1204, 75-127 (2020).

97. McShane, A. et al. Mucus. Curr. Biol. 31, R938-R945 (2021).

98. Ridley, C. \& Thornton, D. J. Mucins: the frontline defence of the lung. Biochem Soc. Trans. 46, 1099-1106 (2018).

99. Roy, M. G. et al. Muc5b is required for airway defence. Nature 505, 412-416 (2014).

100. Barbier, D. et al. Influenza A induces the major secreted airway mucin MUC5AC in a protease-EGFR-extracellular regulated kinase-Sp1-dependent pathway. Am. J. Respir. Cell Mol. Biol. 47, 149-157 (2012).

101. Ehre, C. et al. Overexpressing mouse model demonstrates the protective role of Muc5ac in the lungs. Proc. Natl Acad. Sci. USA. 109, 16528-16533 (2012).

102. Evans, C. M. et al. The polymeric mucin Muc5ac is required for allergic airway hyperreactivity. Nat. Commun. 6, 6281 (2015).
103. Gill, S. K. et al. Increased airway glucose increases airway bacterial load in hyperglycaemia. Sci. Rep. 6, 27636 (2016).

104. Baker, E. H. \& Baines, D. L. Airway glucose homeostasis: a new target in the prevention and treatment of pulmonary infection. Chest 153, 507-514 (2018).

105. Baker, E. H. et al. Hyperglycemia and cystic fibrosis alter respiratory fluid glucose concentrations estimated by breath condensate analysis. J. Appl. Physiol. (1985) 102, 1969-1975 (2007).

106. O'Neill, L. A. J. \& Pearce, E. J. Immunometabolism governs dendritic cell and macrophage function. J. Exp. Med. 213, 15-23 (2016).

107. Van den Bossche, J., O'Neill, L. A. \& Menon, D. Macrophage immunometabolism: where are we (going)? Trends Immunol. 38, 395-406 (2017).

108. Mallia, P. et al. Role of airway glucose in bacterial infections in patients with chronic obstructive pulmonary disease. J. Allergy Clin. Immunol. 142, 815-823.e6 (2018).

109. Garnett, J. P. et al. Hyperglycaemia and Pseudomonas aeruginosa acidify cystic fibrosis airway surface liquid by elevating epithelial monocarboxylate transporter 2 dependent lactate-H+ secretion. Sci. Rep. 6, 37955 (2016).

110. Ho, W. E. et al. Metabolomics reveals altered metabolic pathways in experimental asthma. Am. J. Respir. Cell Mol. Biol. 48, 204-211 (2013).

111. Ogger, P. P. \& Byrne, A. J. Macrophage metabolic reprogramming during chronic lung disease. Mucosal Immunol. 14, 282-295 (2021).

112. Pisu, D., Huang, L., Grenier, J. K. \& Russell, D. G. Dual RNA-Seq of Mtb-infected macrophages in vivo reveals ontologically distinct host-pathogen interactions. Cell Rep. 30, 335-350.e4 (2020).

113. Martinez-Moczygemba, M. et al. Pulmonary alveolar proteinosis caused by deletion of the GM-CSFRalpha gene in the $X$ chromosome pseudoautosomal region 1. J. Exp. Med. 205, 2711-2716 (2008).

114. Shibata, Y. et al. GM-CSF regulates alveolar macrophage differentiation and innate immunity in the lung through PU.1. Immunity 15, 557-567 (2001).

115. Suzuki, T. et al. Pulmonary macrophage transplantation therapy. Nature 514, 450-454 (2014).

116. Willinger, T. et al. Human IL-3/GM-CSF knock-in mice support human alveolar macrophage development and human immune responses in the lung. Proc. Natl Acad. Sci. USA. 108, 2390-2395 (2011).

117. Gschwend, J. et al. Alveolar macrophages rely on GM-CSF from alveolar epithelial type 2 cells before and after birth. J. Exp. Med. 218, e20210745 (2021).

118. Cohen, $M$. et al. Lung single-cell signaling interaction map reveals basophil role in macrophage imprinting. Cell 175, 1031-1044.e18 (2018).

119. Croxford, A. L. et al. The Cytokine GM-CSF drives the inflammatory signature of CCR2 + monocytes and licenses autoimmunity. Immunity 43, 502-514 (2015).

120. Eddy, W. E. et al. Stat5 is required for cd103+ dendritic cell and alveolar macrophage development and protection from lung injury. J. Immunol. 198, 4813-4822 (2017).

121. Morris, D. G. et al. Loss of integrin alpha(v)beta6-mediated TGF-beta activation causes Mmp12-dependent emphysema. Nature 422, 169-173 (2003).

122. Koth, L. L. et al. Integrin beta6 mediates phospholipid and collectin homeostasis by activation of latent TGF-beta1. Am. J. Respir. Cell Mol. Biol. 37, 651-659 (2007).

123. Branchett, W. J. et al. Airway macrophage-intrinsic TGF- $\beta 1$ regulates pulmonary immunity during early-life allergen exposure. J. Allergy Clin. Immunol. 147, 1892-1906 (2021).

124. $\mathrm{Yu}, \mathrm{X}$. et al. The cytokine TGF- $\beta$ promotes the development and homeostasis of alveolar macrophages. Immunity 47, 903-912.e4 (2017).

125. McCowan, J. et al. The transcription factor EGR2 is indispensable for tissuespecific imprinting of alveolar macrophages in health and tissue repair. Sci. Immunol. 6, eabj2132 (2021).

126. Minutti, C. M. et al. A macrophage-pericyte axis directs tissue restoration via amphiregulin-induced transforming growth factor beta activation. Immunity $\mathbf{5 0 \text { , }}$ 645-654.e6 (2019).

127. Snelgrove, R. J. et al. A critical function for CD200 in lung immune homeostasis and the severity of influenza infection. Nat. Immunol. 9, 1074-1083 (2008).

128. Baker, A. D. et al. PPARgamma regulates the expression of cholesterol metabolism genes in alveolar macrophages. Biochem Biophys. Res Commun. 393, 682-687 (2010).

129. Gautier, E. L. et al. Systemic analysis of PPARy in mouse macrophage populations reveals marked diversity in expression with critical roles in resolution of inflammation and airway immunity. J. Immunol. 189, 2614-2624 (2012).

130. Okreglicka, K. et al. PPARy is essential for the development of bone marrow erythroblastic island macrophages and splenic red pulp macrophages. J. Exp. Med. 218, e20191314 (2021).

131. Szigeti, K. et al. Functional, histopathologic and natural history study of neuropathy associated with EGR2 mutations. Neurogenetics 8, 257-262 (2007).

132. Cain, D. W. et al. Identification of a tissue-specific, C/EBP $\beta$-dependent pathway of differentiation for murine peritoneal macrophages. J. Immunol. 191, 4665-4675 (2013). 
133. Rauschmeier, R. et al. Bhlhe 40 and Bhlhe 41 transcription factors regulate alveolar macrophage self-renewal and identity. EMBO J. 38, e101233 (2019).

134. Imperatore, F. et al. SIRT1 regulates macrophage self-renewal. EMBO J. https:// doi.org/10.15252/embj.201695737 (2017).

135. Nakamura, A. et al. Transcription repressor Bach2 is required for pulmonary surfactant homeostasis and alveolar macrophage function. J. Exp. Med. 210, 2191-2204 (2013).

136. Schlitzer, A. et al. IRF4 transcription factor-dependent CD11b+ dendritic cells in human and mouse control mucosal IL-17 cytokine responses. Immunity 38, 970-983 (2013).

137. Wang, Y. et al. IL-34 is a tissue-restricted ligand of CSF1R required for the development of Langerhans cells and microglia. Nat. Immunol. 13, 753-760 (2012).

138. Bellomo, A. et al. Reticular fibroblasts expressing the transcription factor WT1 define a stromal niche that maintains and replenishes splenic red pulp macrophages. Immunity 53, 127-142.e7 (2020).

139. Mondor, I. et al. Lymphatic endothelial cells are essential components of the subcapsular sinus macrophage niche. Immunity 50, 1453-1466.e4 (2019).

140. Albers, G. J. et al. IRF5 regulates airway macrophage metabolic responses. Clin. Exp. Immunol. 204, 134-143 (2021).

141. Zhou, B. et al. The angiocrine Rspondin3 instructs interstitial macrophage transition via metabolic-epigenetic reprogramming and resolves inflammatory injury. Nat. Immunol. 21, 1430-1443 (2020).

142. Keerthivasan, S. et al. Homeostatic functions of monocytes and interstitial lung macrophages are regulated via collagen domain-binding receptor LAIR1. Immunity 54, 1511-1526.e8 (2021).

143. Huh, D., Torisawa, Y.-S., Hamilton, G. A., Kim, H. J. \& Ingber, D. E. Microengineered physiological biomimicry: organs-on-chips. Lab Chip 12, 2156-2164 (2012).

144. Bhatia, S. N. \& Ingber, D. E. Microfluidic organs-on-chips. Nat. Biotechnol. 32 760-772 (2014).

145. Ahadian, S. et al. Organ-on-a-chip platforms: a convergence of advanced materials, cells, and microscale technologies. Adv. Healthc Mater. 7, https://doi. org/10.1002/adhm.201700506 (2018).

146. Zamprogno, P. et al. Second-generation lung-on-a-chip with an array of stretchable alveoli made with a biological membrane. Commun. Biol. 4, 168-10 (2021).

147. Leibel, S. L., McVicar, R. N., Winquist, A. M. \& Snyder, E. Y. Generation of 3D whole lung organoids from induced pluripotent stem cells for modeling lung developmental biology and disease. J. Vis. Exp. e62456 https://doi.org/10.3791/62456 (2021).

148. Miller, A. J. et al. Generation of lung organoids from human pluripotent stem cells in vitro. Nat. Protoc. 14, 518-540 (2019).

149. Coward, W. R., Saini, G. \& Jenkins, G. The pathogenesis of idiopathic pulmonary fibrosis. Ther. Adv. Respir. Dis. 4, 367-388 (2010)

150. Martinez, F. J. et al. Idiopathic pulmonary fibrosis. Nat. Rev. Dis. Prim. 3, 17074-19 (2017).

151. Vijayakumar, B. et al. CT Lung abnormalities after COVID-19 at 3 months and 1 year after hospital discharge. Radiology 211746 https://doi.org/10.1148/ radiol.2021211746 (2021).

152. Byrne, A. J., Maher, T. M. \& Lloyd, C. M. Pulmonary macrophages: a new therapeutic pathway in fibrosing lung disease? Trends Mol. Med. https://doi.org/ 10.1016/j.molmed.2016.02.004 (2016).

153. Hirani, N. et al. Target inhibition of galectin-3 by inhaled TD139 in patients with idiopathic pulmonary fibrosis. Eur. Respir. J. 57, (2021).

154. Joshi, N. et al. A spatially restricted fibrotic niche in pulmonary fibrosis is sustained by M-CSF/M-CSFR signalling in monocyte-derived alveolar macrophages. Eur. Respir. J. 55, 1900646 (2020).

155. Reyfman, P. A. et al. Single-cell transcriptomic analysis of human lung provides insights into the pathobiology of pulmonary fibrosis. Am. J. Respir. Crit. Care Med. 199, 1517-1536 (2019).

156. Aran, D. et al. Reference-based analysis of lung single-cell sequencing reveals a transitional profibrotic macrophage. Nat. Immunol. 20, 163-172 (2019).

157. Humphries, D. C. et al. Selective myeloid depletion of galectin-3 offers protection against acute and chronic lung injury. Front Pharm. 12, 715986 (2021).

158. Berman, J. S. et al. Altered bleomycin-induced lung fibrosis in osteopontindeficient mice. Am. J. Physiol. Lung Cell Mol. Physiol. 286, L1311-L1318 (2004).

159. Adams, T. S. et al. Single-cell RNA-seq reveals ectopic and aberrant lung-resident cell populations in idiopathic pulmonary fibrosis. Sci Adv. 6, eaba1983 (2020).

160. Ucero, A. C. et al. Fra-2-expressing macrophages promote lung fibrosis in mice. J. Clin. Invest. 129, 3293-3309 (2019).

161. Brody, S. L. et al. Chemokine receptor 2-targeted molecular imaging in pulmonary fibrosis. A clinical trial. Am. J. Respir. Crit. Care Med. 203, 78-89 (2021).

162. Gibbons, M. A. et al. Ly6Chi monocytes direct alternatively activated profibrotic macrophage regulation of lung fibrosis. Am. J. Respir. Crit. Care Med. 184, 569-581 (2011)

163. Osterholzer, J. J. et al. Implicating exudate macrophages and Ly-6C(high) monocytes in CCR2-dependent lung fibrosis following gene-targeted alveolar injury. J. Immunol. 190, 3447-3457 (2013).
164. Okuma, T. et al. C-C chemokine receptor 2 (CCR2) deficiency improves bleomycin-induced pulmonary fibrosis by attenuation of both macrophage infiltration and production of macrophage-derived matrix metalloproteinases. $J$. Pathol. 204, 594-604 (2004).

165. McCubbrey, A. L. et al. Deletion of c-FLIP from CD11bhi macrophages prevents development of bleomycin-induced lung fibrosis. Am. J. Respir. Cell Mol. Biol. 58, 66-78 (2018).

166. Satoh, T. et al. Identification of an atypical monocyte and committed progenitor involved in fibrosis. Nature 541, 96-101 (2017).

167. Yáñez, A. et al. Granulocyte-monocyte progenitors and monocyte-dendritic cell progenitors independently produce functionally distinct monocytes. Immunity 47, 890-902.e4 (2017).

168. Habermann, A. C. et al. Single-cell RNA sequencing reveals profibrotic roles of distinct epithelial and mesenchymal lineages in pulmonary fibrosis. Sci. Adv. 6, eaba1972 (2020).

169. Cheresh, P. et al. The Sphingosine Kinase 1 Inhibitor, PF543, Mitigates pulmonary fibrosis by reducing lung epithelial cell mtDNA damage and recruitment of fibrogenic monocytes. Int. J. Mol. Sci. 21, 5595, https://doi.org/10.3390/ ijms21165595 (2020).

170. Thannickal, V. J. et al. Blue journal conference. Aging and susceptibility to lung disease. Am. J. Respir. Crit. Care Med. 191, 261-269 (2015).

171. Raghu, G., Weycker, D., Edelsberg, J., Bradford, W. Z. \& Oster, G. Incidence and prevalence of idiopathic pulmonary fibrosis. Am. J. Respir. Crit. Care Med. 174, 810-816 (2006).

172. Watanabe, S. et al. Resetting proteostasis with ISRIB promotes epithelial differentiation to attenuate pulmonary fibrosis. Proc. Natl. Acad. Sci. USA 118, e2101100118 (2021).

173. Bain, C. C. et al. Rate of replenishment and microenvironment contribute to the sexually dimorphic phenotype and function of peritoneal macrophages. Sci. Immunol. 5, eabc4466 (2020).

174. Wang, J. \& Kubes, P. A reservoir of mature cavity macrophages that can rapidly invade visceral organs to affect tissue repair. Cell 165, 668-678 (2016).

175. Deniset, J. F. et al. Gata6+ pericardial cavity macrophages relocate to the injured heart and prevent cardiac fibrosis. Immunity 51, 131-140.e5 (2019).

176. Jin, $\mathrm{H}$. et al. Genetic fate-mapping reveals surface accumulation but not deep organ invasion of pleural and peritoneal cavity macrophages following injury. Nat. Commun. 12, 2863 (2021).

177. Zhang, N. et al. Myeloid-specific blockade of Notch signaling alleviates murine pulmonary fibrosis through regulating monocyte-derived Ly6clo MHCllhi alveolar macrophages recruitment and TGF- $\beta$ secretion. FASEB J. 34, 11168-11184 (2020).

178. Frangogiannis, N. Transforming growth factor- $\beta$ in tissue fibrosis. J. Exp. Med. 217, e20190103 (2020).

179. Sun, T. et al. TGF $\beta 2$ and TGF $\beta 3$ isoforms drive fibrotic disease pathogenesis. Sci. Transl. Med. 13, eabe0407 (2021).

180. Atabai, K. et al. Mfge8 diminishes the severity of tissue fibrosis in mice by binding and targeting collagen for uptake by macrophages. J. Clin. Invest. 119, 3713-3722 (2009)

181. Cui, H. et al. Monocyte-derived alveolar macrophage apolipoprotein E participates in pulmonary fibrosis resolution. JCI Insight 5, e134539 (2020).

182. Huang, S. et al. Macrophage PPAR- $\gamma$ suppresses long-term lung fibrotic sequelae following acute influenza infection. PLOS ONE 14, e0223430 (2019).

183. Ogger, P. P. et al. Itaconate controls the severity of pulmonary fibrosis. Sci. Immunol. 5, eabc1884 (2020).

\section{ACKNOWLEDGEMENTS}

CCB is funded by a Sir Henry Dale Fellowship from the Wellcome Trust/Royal Society (206234/Z/17/Z). ASM is funded by the Medical Research Council UK. Images from Servier Medical Art (smart.servier.com) were used in some of the figures.

\section{COMPETING INTERESTS}

The authors declare no competing interests.

\section{ADDITIONAL INFORMATION}

Correspondence and requests for materials should be addressed to Calum C. Bain or Andrew S. MacDonald.

Reprints and permission information is available at http://www.nature.com/ reprints

Publisher's note Springer Nature remains neutral with regard to jurisdictional claims in published maps and institutional affiliations. 
Open Access This article is licensed under a Creative Commons Attribution 4.0 International License, which permits use, sharing, adaptation, distribution and reproduction in any medium or format, as long as you give appropriate credit to the original author(s) and the source, provide a link to the Creative Commons license, and indicate if changes were made. The images or other third party material in this article are included in the article's Creative Commons license, unless indicated otherwise in a credit line to the material. If material is not included in the article's Creative Commons license and your intended use is not permitted by statutory regulation or exceeds the permitted use, you will need to obtain permission directly from the copyright holder. To view a copy of this license, visit http://creativecommons. org/licenses/by/4.0/.

(c) The Author(s) 2022 